\title{
Small Cell Carcinoma of the Uterine Cervix Misdiagnosed as Squamous Cell Carcinoma
}

\author{
Aymen Lagha ${ }^{1}$, Nadia Bouzid², Samia Kanoun Belajouza², Soumaya Labidi ${ }^{1}$, \\ Asma Saidi², Hamouda Boussen ${ }^{1} \&$ Noureddine Bouaouina ${ }^{2}$ \\ ${ }^{1}$ Department of medical oncology, Abderrahmane Mami Hospital, Ariana, Tunisia \\ ${ }^{2}$ Department of radiotherapy, Sousse, Tunisia \\ Correspondence: Aymen Lagha, MD, Department of Medical Oncology, Abderrahmane Mami Hospital, Rue de \\ l'Hôpital, Ariana 2080, Tunisia. Tel: 216-2444-0097. E-mail: aymen.lagha@hotmail.com
}

Received: September 27, 2012 Accepted: October 15, 2012 Online Published: December 15, 2012

doi:10.5539/cco.v2n1p36 URL: http://dx.doi.org/10.5539/cco.v2n1p36

No competing financial conflicts exist

\begin{abstract}
Small cell carcinoma of the uterine cervix is a rare tumor. It presents clinical and biological features of both cervical neoplasm such as local aggressiveness, and small cell cancer such as early spread and metastases. To date, an effective treatment protocol is not well established. Case: On March 2009, a 44-year-old pregnant woman with small cell carcinoma of the cervix misdiagnosed initially as squamous cell carcinoma, was treated with chemoradiation followed by surgery and brachytherapy. Twenty-three months later, she experienced brain metastases. A metastasectomy lead to an accurate diagnosis. Palliative whole brain radiotherapy was conducted. The patient died of evolutive disease on August 2012. Conclusion: The optimal therapeutic approach of small cell carcinoma of the cervix is still to define. Further studies regarding multimodal treatment are necessary to achieve a significant survival benefit.
\end{abstract}

Keywords: uterine cervix, small cell carcinoma, treatment

\section{Introducation}

Small-cell neuroendocrine cervical carcinoma (SCNCC) is a rare malignancy that represents less than 5\% of all cervical malignancies (Conner, 2002; Abeler, 1994). Its histology and clinical behavior are comparable to that of small cell carcinoma (SCC) of the lung (Korcum, 2008). SCNCC is characterized by an aggressive clinical course and poor prognosis due to the high incidence of lymphatic involvement and hematogenous dissemination occurring at an early stage (Korcum, 2008; Abeler, 1994; Hoskins, 1995). Therefore, the prognosis of SCNCC is worse when compared to the other cervical malignancies. The treatment is based on surgery, radiotherapy and chemotherapy either alone or in combination (Korcum, 2008; Huang, 2006), but a standard treatment protocol is still to define due to the rarity of this entity. Herein, we report a case of SCNCC misdiagnosed initially as squamous cell carcinoma, and review and discuss the clinical course and the modalities of treatment.

\section{Case}

On March 2009, a 44-year-old woman presented with abnormal vaginal bleeding at 29 weeks pregnant. Her past medical history was not significant, and she had never undergone a Pap smear. On gynecologic examination, a mass was noted on the cervix about $5 \mathrm{~cm}$ in size with contact bleeding. The parametrium and pelvic sidewalls were normal. A cervical biopsy demonstrated squamous cell carcinoma of the uterine cervix. A complete workup including cystoscopy, proctosigmoidoscopy and chest X-ray did not show any other abnormal findings. According to the 2002 FIGO staging system, the tumor was classified as stage IB2. The patient underwent cesarean delivery at 34 weeks of pregnancy, and then she received concomitant radio-chemotherapy (1.8 Gy/day, total 45 Gy with weekly cisplatin $40 \mathrm{mg} / \mathrm{m}^{2}$ ) followed by radical colpo-hysterectomy with pelvic and para-aortic lymph node clearance. The resected specimen was clear of residual tumor.

Following surgery, vaginal barchytherapy was administered using the high-dose-rate brachytherapy. The treatment was well tolerated with no significant side effects. 
The patient did not experience any problem until August 2011, when she presented with a generalized tonic-clonic seizures with loss of consciousness. A right proportional hemiplegia with aphasia was recognized. The cerebral computed tomography (CT) scan obtained immediately after the crisis, revealed a mass in her left parietal lobe.

A metastasectomy was performed. Histologically, the cells were small with oval to spindle-shaped hyperchromatic nuclei. No areas of squamous or adenocarcinoma were identified. On immunohistochemical study, the tissue stained negative for cytokeratin 5/6 and positive for synaptophysin and chromogranin $\mathrm{A}$. The final pathologic diagnosis was small cell neuroendocrine carcinoma. A re-examination with immunohistochemical study of histology slides from the initial cervical biopsies lead to an accurate diagnosis: small cell neuroendocrine cervical carcinoma (SCNCC). Palliative treatment based on corticosteroid, and whole brain radiotherapy $30 \mathrm{~Gy}$ in ten fractions was administered. The patient's general condition improved temporarily, but she died of evolutive disease on August 2012, only 12 months after diagnosis of cerebral metastasis.

\section{Discussion}

The uterine cervix is the most common site in the female genital tract for the occurrence of small cell carcinoma which accounts for up to $2 \%$ of all cervical carcinomas (Korcum, 2008; Gardner, 2011). The etiology and pathogenesis of SCNCC are unknown; some reports suggest that it might be strongly associated with human papilloma virus type 18 or 16 or with a high frequency of mutation of the p53 tumor suppressor gene (Korcum, 2008). Median age of diagnosis is in the fifth decade (Gardner, 2011). The diagnosis can be made on cervical biopsy but sometimes, as was the case with our patient, the limited amount of tissue obtained may lead to misdiagnosis as poorly differentiated cervical carcinoma and the neuroendocrine component is recognized only on the colpo-hysterectomy speciman (Gardner, 2011).

SCNCC is characterized by high mitotic rate and frequent involvement of lymph-vascular space (Gardner, 2011). Thus, it behaves aggressively with a frequency and manner of recurrence that is unusual for other types of cervical cancer (Viswanathan, 2004). In fact, these tumors behave as their counterparts of the lung (Gardner, 2011), and they tend toward rapid extrapelvic distant metastases to areas such as the lung, liver, brain, bone and lymph nodes (Nasu, 2011). Given this high rate of distant metastatic, PET/CT imaging may be a helpful tool to detect early metastases (Gardner, 2011). However, Head CT is not performed on initial evaluation for SCNCC due to the low incidence of initial brain metastases (Hoskins, 2003; Gardner, 2011).

Owing to its aggressive nature, SCNCC have a prognosis that is significantly poorer than that for stage-comparable poorly differentiated squamous cell carcinoma of the uterine cervix (Gardner, 2011). The overall 5-year survival rate for patients with SCNCC has been reported to range from $17 \%$ to $67 \%$ for early stage disease, and from 0 to 17\% for advanced stage (Nasu, 2011; Bifulco, 2009). More specifically, the 5-year survival rate of patients with FIGO stage IB1 disease is between $50 \%$ and $60 \%$, which is much worse than the $90 \%$ rate for stage IB1 squamous cell carcinoma (Viswanathan, 2004). The clinical and pathological prognostic factors recognized to have poor prognosis are: smoking status, polypoid pattern, presence of positive lymph nodes, pure small cell histology, treatment with surgery, margin status and advanced FIGO stage (Gardner, 2011; Nasu, 2011; Bifulco, 2009).

Due to the low incidence of SCNCC, no multicenter study has been conducted and the initial standard management of this disease is not well defined. Therefore, the treatment of SCNCC takes into account both treatment options for cervical squamous cell cancer and small cell lung cancer at the same time. One important point is that pelvic control alone is not usually enough to reach a better survival rate due to the early spread of the disease (Boruta, 2001; Korcum, 2008; Bifulco, 2009), thus controlling hematogenous spread is and should be a necessity (Bifulco, 2009). A multidisciplinary therapy, including surgery, chemotherapy and radiotherapy is mandatory (Korcum, 2008; Bifulco, 2009; Gardner, 2011; Nasu, 2011).

Surgery is based on radical colpohysterectomy with regional lymphadenectomy (Gardner, 2011; Bifulco, 2009), but it is associated with morbidities such as lymphedema, chronic bladder dysfunction, ureterovaginal or vesicovaginal fistula (Bifulco, 2009). As well, the delay in systemic adjuvant treatment resulting from even a short postsurgical recovery period might place a patient at risk for metastatic spread (Nasu, 2011; Bifulco, 2009). Thus, the neoadjuvant chemotherapy may represent a reasonable option in the management of SCNCC (Lewandowski, 1993; Nasu, 2011). Also, adjuvant chemotherapy provides a significant survival advantage for patients either with or without lymph node metastasis (Gardner, 2011). Chemotherapy regimens are extrapolated from those used in small cell carcinoma of the lung, and are based either on vincristine/adriamycin/ cyclophosphamide (VAC) or cisplatin/etoposide (EP) (Chang, 1998; Boruta, 2001). However, EP regimens are 
more commonly used due to their lower toxicity when compared to the VAC regimens (Roth, 1992; Gardner, 2011). In lung cancers, concurrent chemoradiation is standard treatment for localized small cell carcinoma, leading some authors to consider this modality a reasonable option in SCNCC (Hoskins, 2003).

Since the treatment of SCNCC is extrapolated from small neuroendocrine carcinomas of the lung, one important question is the place of prophylactic brain irradiation (WBRT). Hoskins et al. initially used WBRT, but dropped it due to the rarity of recurrence in the brain (Hoskins, 2003). Moreover, it has been reported that brain metastases were only present with lung metastases (Viswanathan, 2004); thus prophylactic brain irradiation is not performed routinely, and Head CT scan is only performed whenever lung metastases are documented (Gardner, 2011). In the presented case, the patient experienced brain metastases without any evidence of other distant metastases.

Recently, Gardner et al. (2011) proposed in 2011 a management algorithm for SCNCC: radical hysterectomy and lymphadenectomy with consideration of EP regimen as adjuvant chemotherapy for tumors less than $4 \mathrm{~cm}$. When tumors are larger than $4 \mathrm{~cm}$, a neoadjuvant chemotherapy can be considered to enhance the resecability of the bulky tumors, followed by a localized treatment if the disease remains limited. In late stage or non-surgical candidates, combination chemotherapy with RT for local control remains a reasonable approach.

\section{Conclusion}

Small cell carcinoma of the uterine cervix is characterized by its rarity but as well by its local and distant aggressiveness. The prognosis is still poor due to the frequent and early metastases. Immunohistochemistry is recommended to achieve the accurate diagnosis of SCNCC in order to start the appropriate treatment. However, the optimal therapeutic approach is not well defined and is still controversial. Locoregional treatment alone is insufficient in most patients to achieve a good outcome. In order to improve the survival rates, SCNCC requires a multi-modality therapeutic approach involving systemic treatment to effectively control distant metastases, and surgery or radiotherapy to effectively control local and regional disease. A well-designed multicenter clinical trials are needed to define the best modality of treatment.

\section{References}

Conner, M. G., Richter, H., Moran, C. A., Hameed, A., \& Albores-Saavedra, J. (2002). Small cell carcinoma of the cervix: A clinicopathologic and immunohistochemical study of 23 cases. Ann Diagn Pathol, 6, 345-348. http://dx.doi.org/10.1053/adpa.2002.36661

Abeler, V. M., Holm, R., Nesland, J. M., \& Kjorstad, K. E. (1994). Small cell carcinoma of the cervix. A clinicopathologic study of 26 patients. Cancer, 73(3), 672-677. http://dx.doi.org/10.1002/1097-0142(19940201)73:3<672::AID-CNCR2820730328>3.0.CO;2-R

Korcum, A. F., Aksu, G., Bozcuk, H., Pestereli, E., \& Simsek, T. (2008). Small cell carcinoma of the cervix: a case report. Arch Gynecol Obstet, 277, 367-370. http://dx.doi.org/10.1007/s00404-007-0463-9

Huang, L. C., Li, C. P., Chen, C. C., et al. (2006). Small Cell Carcinoma of the Uterine Cervix: A Case Report with Emphasis on Cytopathological Findings. Tzu Chi Med J, 18(6), 456-461.

Hoskins, P. J., Wong, F., Swenerton, K. D., Pike, J. A., Manji, M., McMurtrie, E., et al. (1995). Small cell carcinoma of the cervix treated with concurrent radiotherapy, cisplatin, and etoposide. Gynecol Oncol, 56(2), 218-225. http://dx.doi.org/10.1006/gyno.1995.1035

Gardner, J. G., Reidy-Lagunes, D., \& Gehrig, P. A. (2011). Neuroendocrine tumors of the gynecologic tract: a society of gynecologic oncology (SGO) clinical document. Gynecolgic oncology, 122, 190-198. http://dx.doi.org/10.1016/j.ygyno.2011.04.011

Viswanathan, A. N., Deavers, M. T., Jhingran, A., Ramirez, P. T., Levenback, C., \& Eifel, P. J. (2004). Small cell neuroendocrine carcinoma of the cervix: outcome and patterns of recurrence. Gynecologic oncology, 93, 27-33. http://dx.doi.org/10.1016/j.ygyno.2003.12.027

Nasu, K., Hirakawa, T., Okamoto, M., et al. (2011). Advanced small cell carcinoma of the uterine cervix treated by neoadjuvant chemotherapy with irinotecan and cisplatin followed by radical surgery. Rare tumors, 3 , 18-20.

Bifulco, G., Mandato, V. D., Giampaolino, P., et al. (2009). Small cell neuroendocrine cervical carcinoma with 1-year follow-up: case report and review. Anticancer research, 29, 477-484.

Hoskins, P. J., Swenerton, K. D., Pike, J. A., Lim, P., Aquino-Parsons, C., Wong, F., et al. (2003). Small-cell carcinoma of the cervix: fourteen years of experience at a single institution using a combined-modality 
regimen of involved-field irradiation and platinum-based combination chemotherapy. J Clin Oncol, 21, 3495-501. http://dx.doi.org/10.1200/JCO.2003.01.501

Boruta, D. M. II., Schorge, J. O., Duska, L. A., Crum, C. P., Castrillon, D. H., \& Sheets, E. E. (2001). Multimodality therapy in early-stage neuroendocrine carcinoma of the uterine cervix. Gynecol Oncol, 81(1), 82-87. http://dx.doi.org/10.1006/gyno.2000.6118

Chang, T. C., Lai, C. H., Tseng, C. J., Hsueh, S., Huang, K. G., \& Chou, H. H. (1998). Prognostic factors in surgically treated small cell cervical carcinoma followed by adjuvant chemotherapy. Cancer, 83, 712-8. http://dx.doi.org/10.1002/(SICI)1097-0142(19980815)83:4<712::AID-CNCR12>3.0.CO;2-V

Roth, B. J., Johnson, D. H., Einhorn, L. H., Schacter, L. P., Cherng, N. C., Cohen, H. J., et al. (1992). Randomized study of cyclophosphamide, doxorubicin, and vincristine versus etoposide and cisplatin versus alternation of these two regimens in extensive small-cell lung cancer: a phase III trial of the Southeastern Cancer Study Group. J Clin Oncol, 10, 282-91.

Lewandowski, G. S., \& Copeland, L. J. (1993). A potential role for intensive chemotherapy in the treatment of small cell neuroendocrine tumors of the cervix. Gynecol Oncol, 48, 127-31. http://dx.doi.org/10.1006/gyno.1993.1021 